\title{
A Log-Dagum Weibull Distribution: Properties and Characterization
}

\author{
Aneeqa Khadim ${ }^{1}$, Aamir Saghir ${ }^{1}$, Tassadaq Hussain ${ }^{1}$, Mohammad Shakil ${ }^{2}$, \\ Mohammad Ahsanullah ${ }^{3}$ \\ ${ }^{1}$ Department of Mathematics, Faculty of Sciences, Mirpur University of Science and Technology (MUST), Mirpur, Pakistan \\ ${ }^{2}$ Department of Liberal Arts and Sciences, Faculty of Mathematics, Miami Dade College, Hialeah, USA \\ ${ }^{3}$ Department of Management Sciences, Rider University, Lawrenceville, USA
}

Email address:

Aneeqa89@gmail.com (A. Khadim)

\section{To cite this article:}

Aneeqa Khadim, Aamir Saghir, Tassadaq Hussain, Mohammad Shakil, Mohammad Ahsanullah. A Log-Dagum Weibull Distribution: Properties and Characterization. Applied and Computational Mathematics. Vol. 10, No. 5, 2021, pp. 100-113. doi: $10.11648 /$ j.acm. 20211005.11

Received: September 10, 2021; Accepted: September 27, 2021; Published: October 12, 2021

\begin{abstract}
This article proposes a new family of continuous distributions generated from a log dagum random variable (named Log-Dagum Weibull Distribution) on the basis of T-X family technique. mathematical and statistical properties including survival function, hazard and reverse hazard function, Rth moments, L-moments, incomplete rth moments, quantile points, Order Statistics, Bonferroni and Lorenz curves as well as entropy measures for this class of distributions are presented also LDW distribution characterized by truncated moments order statistics and upper record values. Simulation study of the proposed family of distribution has been derived. The model parameters are obtained by the method of maximum likelihood estimation. We illustrate the performance of the proposed new family of distributions by means of four real data sets and the data sets show the new family of distributions is more appropriate as compared to Exponentiated exponential distribution (EED), Weibull distribution (WD), Gamma distribution (GD), NEED Nadarajah Exponentiated exponential distribution and Lomax distribution (LD). Moreover, perfection of competing models is also tested via the Kolmogrov-Simnorov (K S), the Anderson Darling $\left(A^{*}\right)$ and the Cramer-von Misses $\left(W^{*}\right)$. The measures of goodness of fit including the Akaike information criterion (AIC), consistent Akaike information criterion (CAIC), Bayesian information criterion (BIC), Hannan-Quinn information criterion (HQIC) are computed to compare the fitted models.
\end{abstract}

Keywords: Probability Distributions, Log-Dagum Distribution, Parameter Estimation, Weibull Distribution

\section{Introduction}

Statistical distributions are extensively used in the literature for the modeling and the forecasting of real life phenomena. The recent literature has suggested numerous ways of extending well-known distributions. There has been an increased interest in defining new classes of the univariate continuous distributions by introducing one or more additional shape parameter(s) to the baseline distribution. This induction of parameter(s) has been proven useful in exploring tail properties and also for improving the goodness-of-fit of the generator family. The well-known families are: The beta-G of Eugene et al. [19] the Gamma-G (type-1) proposed by Zografos and Balakrishnan [35] the Kumaraswamy-G derived by Cordeiro and de Castro [14] the
Mc-G family considered by Alexander et al. [5] the Weibull-X family of distributions developed by Alzaatreh et al. [3] the exponentiated generalized class derived by Cordeiro et al. [15] the Exponentiated T-X family developed by Alzaghal et al. [1] the weibull-G family proposed by Bourguignon et al. [13] the exponentiated half-logistic family considered by Cordeiro et al. [17] the gamma-G (type 3) family introduced by Torabi and Montazari [33] the log-gamma-G family developed by Amini et al. [7] the gamma-x family introduced by Alzaatreh et al. [4] the logistic-G family introduced by Torabi and Montazari [32]. The Kumaraswamy weibull-generated family developed by Hassan and Elgarhy [23] the new weibull-G family discussed by Tahir et al.[34] Exponentiated generalized exponential dagum distribution extended by Nasiru et al [25] the new generalized family of distributions discussed by Ahmad [2] Some new 
members of the T-X family of distributions of Farrukh Jamal and Muhammad Nasir [20]. A modified T-X family of distributions discussed by Muhammad Aslam et al. [6] Handique et al. [22] discussed properties and applications of a new member of the T-X family of distributions, Shakil et al. [31] derived properties of Burr (4P) distribution and Shakil et al. [30] discussed some inferences on the Dagum (4P) distribution. Hamed et al. [21] established new class of Lindley distributions properties and application etc.

The current work presents a new distribution called the Log-Dagum Weibull (LDW) distribution with three parameters. The proposed distribution due to its flexibility in accommodating all the forms of the hazard rate function can be used in a variety of problems for modeling lifetime data. The LDW distribution is not only suitable for modeling comfortable bathtub shaped failure rates data but is also suitable for testing goodness-of-fit of some models.

The rest of the article is organized as follows. Section 2 presents the $\mathrm{T}-\mathrm{X}$ family of distributions. In section 3 , the proposed Log-Dagum Weibull distribution is derived and studied graphical behavior of its probability density functions (pdf), survival function hazard functions shape of hazard function and concavity. In section 4, some statistical properties including rth moments, L- moment's, incomplete rth moments, quantile function and order statistics are presented. Section 5, contains the Shannon entropy and Renyi entropy. Section 6 presents Bonferroni and Lorenz curves. The characterization via hazard function, reverse hazard function and truncated moments and ordered statistics of distribution is derived in section 7. Estimation of model parameters is presented in section 8. Evaluation measures and practical data examples of the proposed model to real data are given in section 9 , followed by concluding remarks.

\section{T-X Family}

Let $r(t)$ be the probability density function (pdf) of a random variable $T \in[a ; b]$ for $-\infty \leq a<b<\infty$ and let $W[G(x)]$ be a function of the cumulative distribution function (cdf) of a randomvariable $X$ such that $W[G(x)]$ satisfies the following conditions

$$
\left\{\begin{array}{c}
\text { (i) } W[G(x)] \in[\mathrm{a}, \mathrm{b}] \\
(i i) W[G(x)] \text { Is differentiable and monotonically non }- \text { decreasing } \\
\text { and }(i i i) \mathrm{W}[\mathrm{G}(\mathrm{x})] \rightarrow \text { a as } \mathrm{x} \rightarrow-\infty \text { and } \mathrm{W}[\mathrm{G}(\mathrm{x})] \in \mathrm{b} \text { as } \mathrm{x} \rightarrow \infty
\end{array}\right.
$$

Recently, Alzaatreh et al. [3] defined the T-X family of distributions by

$$
F(x)=\int_{0}^{W[G(x)} r(t) d t
$$

Where $W[G(x)]$ satisfies the condition (1). The pdf corresponding to (2) is given by

$$
f(x)=\left\{\frac{d}{d x} W[G(x)]\right\} r\{W[\mathrm{G}(\mathrm{x})]\}
$$

In Table 1 , we provide the $\mathrm{W}[\mathrm{G}(\mathrm{x})]$ functions for some members of the T-X family of distributions.

Table 1. Different $W[G(x)]$ functions for special models of the T-X family.

\begin{tabular}{llll}
\hline S.No. & $\boldsymbol{W}[\boldsymbol{G}(\boldsymbol{x})]$ & Range of $\boldsymbol{T}$ & Members of T-X family \\
\hline & & & Beta-G 19] \\
1 & $G(x)$ & {$[0,1]$} & KW-G type 1 [14] \\
& & Mc-G [5] \\
& & Exp-G (KW-G type 2) [15] & Gamma-G Type-2 [35] \\
2 & $-\log [\mathrm{G}(\mathrm{x})]$ & {$[0, \infty]$} & Log-Gamma-G Type-2 [7] \\
& & & Gamma-G Type-1 [26] \\
& & Log-Gamma-G [7] \\
3 & $-\log [1-G(x)]$ & {$[0, \infty]$} & Weibull-X [3] \\
& & & Gamma-X [4] \\
4 & $-\log \left[1-G^{\alpha}(x)\right]$ & Exponentiated T-X [1] \\
5 & $\log [-\log [1-G(x)]$ & {$[-\infty]$} & Logistic-X \\
6 & $\log \left[\frac{\mathrm{G}(\mathrm{x})}{1-\mathrm{G}(\mathrm{x})}\right]$ & {$[-\infty, \infty]$} & Logistic-G [32] proposed \\
7 & $\frac{\mathrm{G}(\mathrm{x})}{1-\mathrm{G}(\mathrm{x})}$ & {$[0, \infty]$} & Gamma-G Type-3 [35] \\
\hline
\end{tabular}

\section{New Model}

A random variable $\mathrm{T}$ has the log dagum distribution with shape parameter $\beta>0$ and $\lambda>0$ if it's cumulative distribution function (cdf) is given by

$$
\pi(t)=\left(1+\mathrm{e}^{-\lambda x}\right)^{-\beta} t \in R, \beta>0, \lambda>0
$$

Its corresponding probability density function (pdf) can be expressed as

$$
r(t)=\beta \lambda \mathrm{e}^{-\lambda x}\left(1+\mathrm{e}^{-\lambda x}\right)^{-\beta-1} t \in R, \beta>0, \lambda>0
$$

Let $G(x)$ and $\vec{G}(x)=1-G(x)$ be the baseline cdf and survival function (sf) by replacing $W[G(x)]$ by $\log \left(\frac{\mathrm{G}(\mathrm{x})}{1-\mathrm{G}(\mathrm{x})}\right)$ and $r(t)$ with (5) in equation (2), we define the cdf of the log dagum-x family by 


$$
F(x)=\left[1+\left(\frac{G(x)}{1-G(x)}\right)^{-\lambda}\right]^{-\beta}
$$

The log dagum family pdf is expressed as

$$
f(x)=\left[1+\left(\frac{G(x)}{1-G(x)}\right)^{-\lambda}\right]^{-\beta-1}\left(\frac{G(x)}{1-G(x)}\right)^{-\lambda-1} \frac{\lambda \beta g(x)}{[1-G(x)]^{2}}
$$

Henceforth, we denote by $X$ a random variable having density function (7). The basic motivations for using the log dagum-x family in practice are to construct heavy tailed distributions that are not longer-tailed for modeling real data, to generate distributions with symmetric, left-skewed, rightskewed and reversed-J shaped, to define special models with all types of the hazard rate function (hrf), to provide consistently better fits than other generated models under the same baseline distribution.

The corresponding cumulative density function (cdf) probability density function (pdf), hazard function (hrf) and survival function are given as

$$
F_{L D W}(x, \lambda, \theta, \beta)=\left(1+\left(\mathrm{e}^{\theta x^{\beta}}-1\right)^{-\lambda}\right)^{-\beta}
$$

a) CDF Plot for LDWD

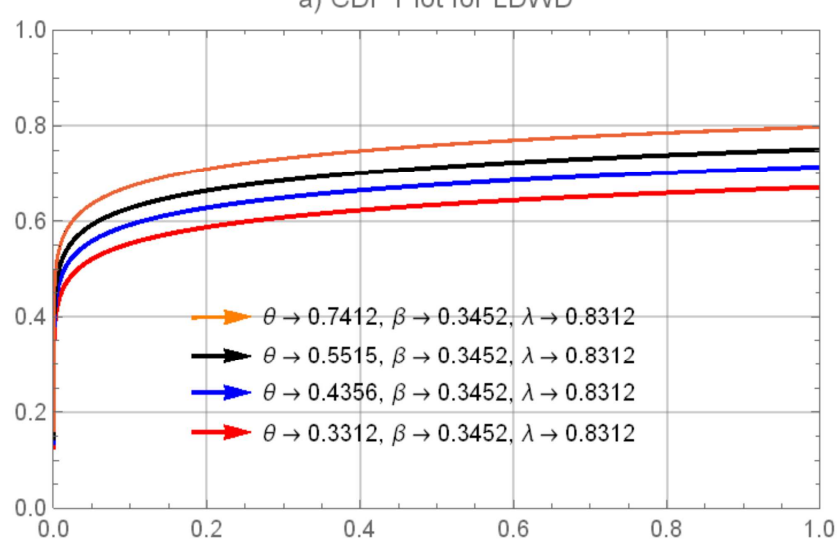

b) CDF Plot for LDWD

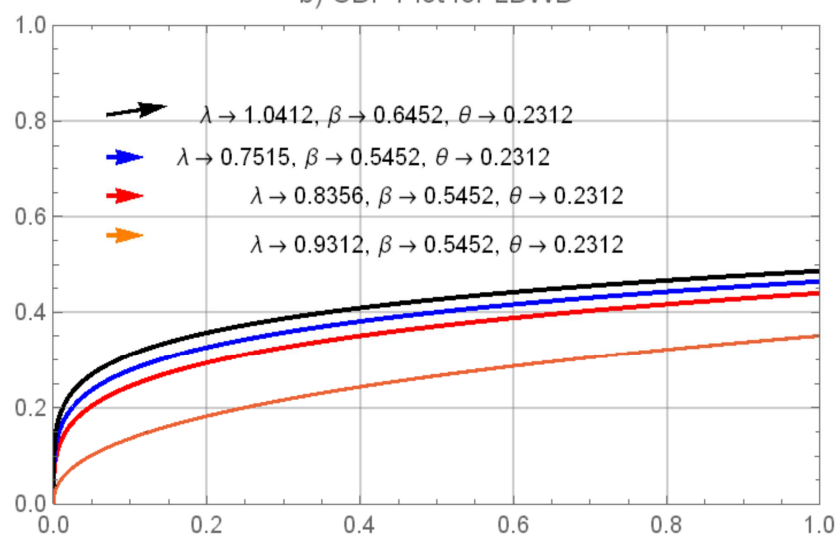

Figure 1. Cumulative distribution plot of $L D W D$.

Figure 1 gives the plots of the cumulative distribution function of the LDW distribution.

The plots of this figure shows that for fixed $\lambda$ and $\beta$ and changing $\theta$ the curve stretch out insignificantly towards right as $\theta$ increases. However, for fixed $\beta$ and $\theta$ and changing $\lambda$.
Thecurve stretches out towards right significantly as $\lambda$ increases.

And

$$
\begin{array}{r}
f_{L D W}(x, \lambda, \theta, \beta)= \\
\left(1+\left(\mathrm{e}^{\theta x^{\beta}}-1\right)^{-\lambda}\right)^{-\beta-1}\left(-1 \mathrm{e}^{\theta x^{\beta}}\right)^{-\lambda-1} \mathrm{e}^{\theta x^{\beta}} \theta \lambda \beta^{2} x^{\beta-1}
\end{array}
$$
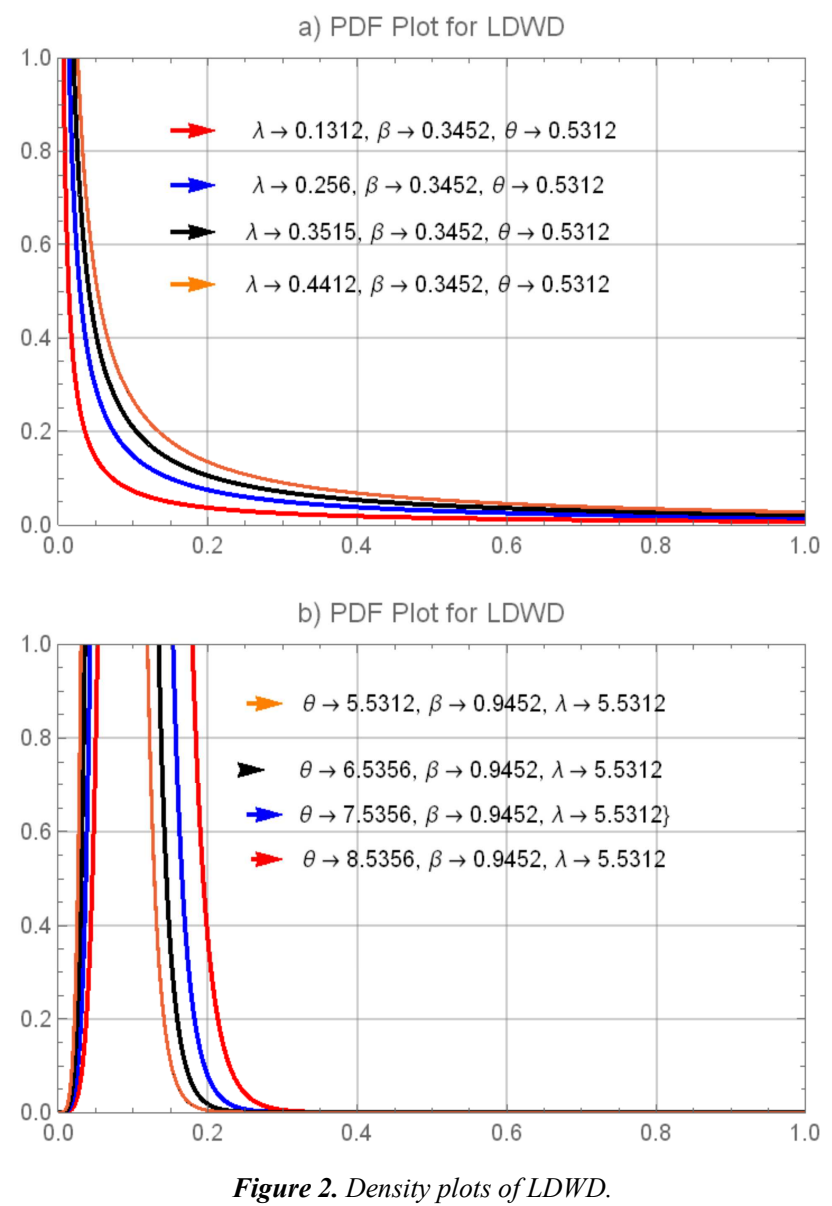

Plots of Figure 2 display the density functions of the LDW distribution. Figure 2 portrays that changing $\lambda$ against the fixed $\beta$ and $\theta$ the density function decreases. But changing $\beta$ against the fixed $\lambda$ the nature of the curve towards right as $\theta$ increases, however in case of changing $\theta$ with fixed $\beta$ and $\lambda$ shift the curve towards left.

$$
S_{L D W}(x, \lambda, \theta, \beta)=1-\left(1+\left(\mathrm{e}^{\theta x^{\beta}}-1\right)^{-\lambda}\right)^{-\beta}
$$

a) survival function Plot for LDWD

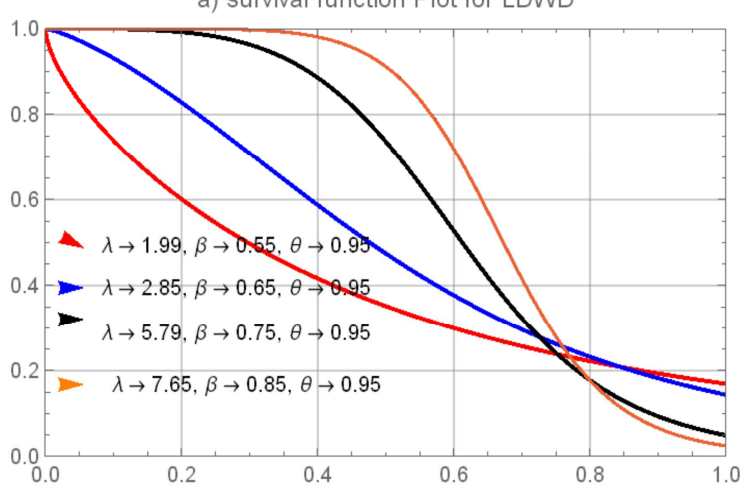


c) survival function Plot for LDWD

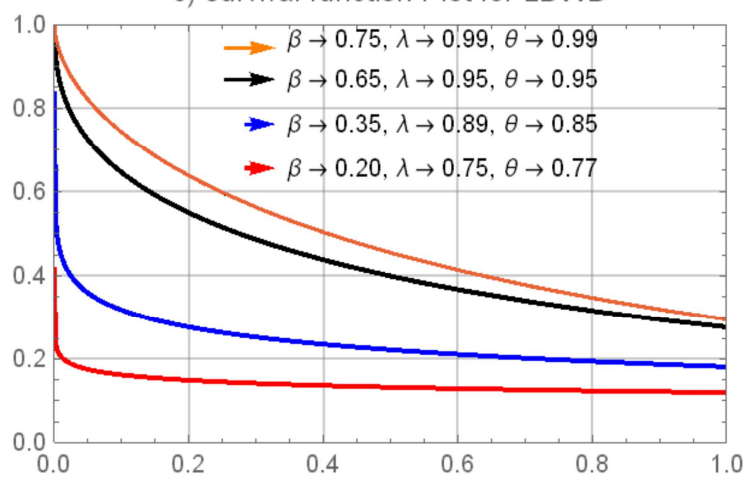

Figure 3. Survival plot of $L D W D$.

The graph of survival function increases for different values of parameters then suddenly starts gradually decreases and converges to zero.

$$
h(x, \lambda, \beta)=\frac{\left(1+\left(e^{\theta x^{\beta}}-1\right)^{-\lambda}\right)^{-\beta-1}\left(-1+e^{\theta x^{\beta}}\right)^{-\lambda-1} e^{\theta x^{\beta}} \theta \lambda \beta^{2} x^{\beta-1}}{1-\left(1+\left(e^{\theta x^{\beta}}-1\right)^{-\lambda}\right)^{-\beta}}
$$

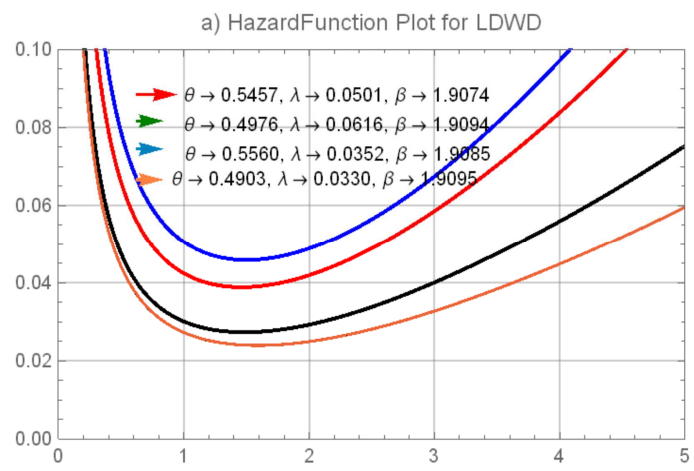

b) HazardFunction Plot for LDWD

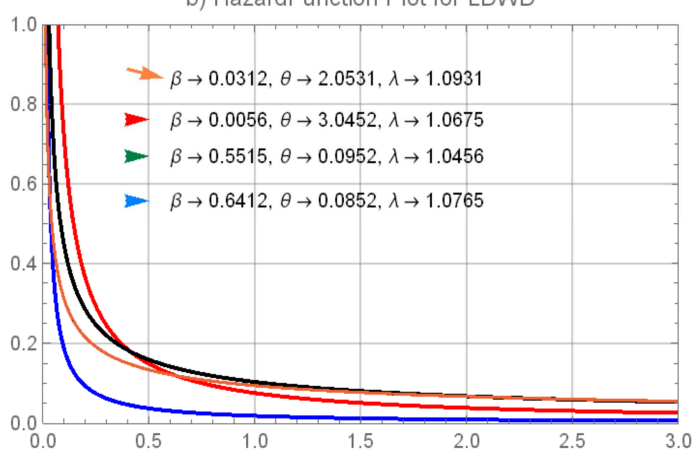

c) HazardFunction Plot for LDWD

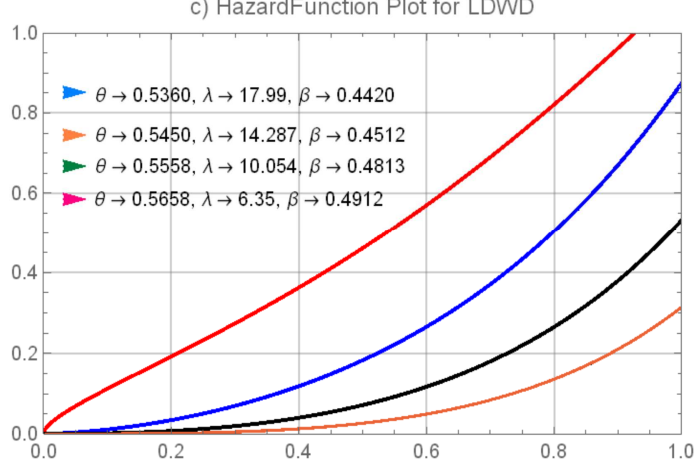

Figure 4. Hazard plot of $L D W D$
The hazard function plots in Figure 4 also portray the declining circumstance of the product as time increases in terms of impulsive spikes at the end of either increasing or decreasing hazard rate. This implies that the hazard function is sensitive against different combinations of the parameters as time changes, which seems to be a refine image of non stationary process and hence the hazard curve does not remain stable as times passes. Moreover, Figure 4 displays increasing, decreasing bathtub hazard shapes.

\subsection{Shape of Hazard Function}

Shape of the density function can be described analytically, the critical point of the LDW density are the root of the equation

$$
\frac{d}{d x}\left[\frac{\left(1+\left(e^{\theta x^{\beta}}-1\right)^{-\lambda}\right)^{-\beta-1}\left(-1+e^{\theta x^{\beta}}\right)^{-\lambda-1} e^{\theta x^{\beta}} \theta \lambda \beta^{2} x^{\beta-1}}{1-\left(1+\left(e^{\theta x \beta}-1\right)^{-\lambda}\right)^{-\beta}}\right]=0
$$

There may be more than one root.

\subsection{Concavity}

The concavity of hazard rate function $h^{\prime \prime}(x)=0$

$$
\frac{\mathrm{d}^{2}}{\mathrm{dx}^{2}}\left[\frac{\left(1+\left(e^{\theta x^{\beta}}-1\right)^{-\lambda}\right)^{-\beta-1}\left(-1+e^{\theta x \beta}\right)^{-\lambda-1} e^{\theta x \beta} \theta \lambda \beta^{2} x^{\beta-1}}{1-\left(1+\left(e^{\theta x^{\beta}}-1\right)^{-\lambda}\right)^{-\beta}}\right]=0
$$

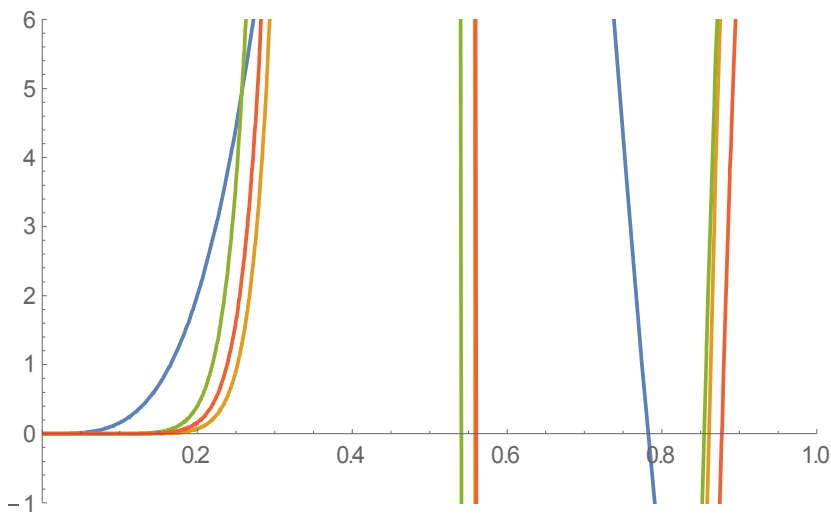

Figure 5. Concavity of different value of parameters the hazard function is concave up and concave down where the point of concavity change is called point of inflection.

\section{Some Statistical Properties}

In this section, we study some statistical properties of the LDW distribution, including Rth moments, L-moments, incomplete rth moments, quantile function and order statistics.

\subsection{Moments of Ldwd}

Let $\mathrm{X}$ is a particularly continuous non-negative random variable with $\operatorname{PDF} f(X)$, and then the $\mathrm{R}^{\text {th }}$ ordinary moment of the (LDW) distribution is given by: 


$$
\begin{gathered}
\mathrm{E}\left(\mathrm{x}^{\mathrm{r}}\right)=\int_{0}^{\infty} \mathrm{X}^{\mathrm{r}} \mathrm{f}(\mathrm{X}) \mathrm{dX} \\
E\left(x^{r}\right)=\int_{0}^{\infty} x^{r}\left(1-\left(1-e^{\theta x^{\beta}}\right)^{-\lambda}\right)^{-\beta-1}\left(1-e^{\theta x^{\beta}}\right)^{-\lambda-1} e^{\theta x^{\beta}} \theta \lambda \beta^{2} x^{\beta-1} d x \mathrm{E}\left(\mathrm{x}^{\mathrm{r}}\right)=\sum_{\mathrm{h}=0}^{\infty} \frac{(-\mathrm{k})_{\mathrm{h}}(-1)^{\mathrm{h}}}{\mathrm{h} !} \int_{0}^{\infty} X^{r}(1- \\
\left.e^{\theta x^{\beta}}\right)^{\lambda \beta-1} e^{\theta x^{\beta}} \theta \lambda \beta^{2} x^{\beta-1} d x
\end{gathered}
$$

which, on substituting $y=e^{\theta x^{\beta}}$ and simplifying, reduces to

$$
\mathrm{E}\left(\mathrm{x}^{\mathrm{r}}\right)=\sum_{\mathrm{h}=0}^{\infty} \frac{(-\mathrm{k})_{\mathrm{h}}(-1)^{\mathrm{h}}}{h !} \sum_{\mathrm{m}=0}^{\lambda \beta-1} \frac{(-1)^{\mathrm{m}}}{m !} \int_{0}^{\infty}(\ln y)^{\frac{r}{\beta}} \mathrm{y}^{\mathrm{m}} \mathrm{dy}
$$

Again substituting and simplifying

$$
\begin{gathered}
E\left(x^{r}\right)=\lambda \beta \frac{1}{\theta^{\frac{r}{\beta}}} \sum_{\mathrm{h}=0}^{\infty} \frac{(-\mathrm{k})_{\mathrm{h}}(-1)^{\mathrm{h}}}{h !} \sum_{\mathrm{m}=0}^{\lambda \beta-1} \frac{(-1)^{\mathrm{m}}}{m !} \int_{0}^{\infty} \frac{g}{(m+1)^{\frac{r}{\beta}}} e^{-g} \frac{d g}{(m+1)} \\
E\left(x^{r}\right)=\lambda \beta \frac{1}{\theta^{\frac{r}{\beta}}} \sum_{\mathrm{h}=0}^{\infty} \frac{(-\mathrm{k})_{\mathrm{h}}(-1)^{\mathrm{h}}}{h !} \sum_{\mathrm{m}=0}^{\lambda \beta-1} \frac{(-1)^{\mathrm{m}}}{m !} \frac{1}{(m+1)^{\frac{r}{\beta^{+1}}}} \Gamma\left(\frac{r}{\beta}+1\right)
\end{gathered}
$$

\subsection{L-Moments}

The L-Moments of the LDW family is defined as

$$
\begin{gathered}
B_{k}=E\left(x\left(F(x)^{k}\right)\right. \\
B_{k}=\int_{0}^{\infty} x\left(1-\left(1-\mathrm{e}^{\theta x^{\beta}}\right)^{-\lambda}\right)^{-\beta k} f(x) d x \\
B_{k}=\int_{0}^{\infty} x\left(1-\left(1-\mathrm{e}^{\theta x^{\beta}}\right)^{-\lambda}\right)^{-\beta k}\left(\left(1-\left(1-\mathrm{e}^{\theta x^{\beta}}\right)^{-\lambda}\right)^{-\beta-1}\left(1-\mathrm{e}^{\theta x^{\beta}}\right)^{-\lambda-1} \mathrm{e}^{\theta x^{\beta}} \theta \lambda \beta^{2} x^{\beta-1} d x\right. \\
B_{k}=\sum_{\mathrm{h}=0}^{\infty} \frac{(-\mathrm{k})_{\mathrm{h}}(-1)^{\mathrm{h}}}{h !} \int_{0}^{\infty} x\left(1-\mathrm{e}^{\theta x^{\beta}}\right)^{\lambda \beta(k+1)-1} \mathrm{e}^{\theta x^{\beta}} \theta \lambda \beta^{2} x^{\beta-1} d x
\end{gathered}
$$

Lete $x^{\theta x^{\beta}}=\mathrm{y}, \beta \theta e^{\theta x^{\beta}} x^{\beta-1} \mathrm{dx}=\mathrm{dy}, x=\frac{1}{\theta^{\frac{1}{\beta}}}(\ln y)^{\frac{1}{\beta}} d y$

When $x \rightarrow 0, y \rightarrow 1$ and $x \rightarrow \infty, y \rightarrow \infty$

$$
B_{k}=\frac{\lambda \beta}{\theta^{\frac{1}{\beta}}} \sum_{\mathrm{h}=0}^{\infty} \frac{(-\mathrm{k})_{\mathrm{h}}(-1)^{\mathrm{h}}}{h !} \int_{0}^{\infty}(\ln y)^{\frac{1}{\beta}}(1-\mathrm{y})^{\lambda \beta(k+1)-1} d y
$$

Expanding $(1-\mathrm{y})^{\lambda \beta(k+1)-1}$ using binomial expension

$$
B_{k}=\frac{\lambda \beta}{\theta^{\frac{1}{\beta}}} \sum_{\mathrm{h}=0}^{\infty} \frac{(-\mathrm{k})_{\mathrm{h}}(-1)^{\mathrm{h}}}{h !} \mathrm{e}^{\mathrm{m}+1} \sum_{\mathrm{k}=0}^{\infty} \sum_{\mathrm{m}=0}^{(\lambda \beta(k+1)-1)}\left(\begin{array}{c}
\lambda \beta(k+1)-1 \\
\mathrm{~m}
\end{array}\right)(-1)^{2 \mathrm{~m}} \int_{1}^{\infty}(\ln y)^{\frac{1}{\beta}}(\mathrm{y})^{\mathrm{m}} d y
$$

After some substitution,

$$
\begin{gathered}
B_{k}=\frac{\lambda \beta}{\theta^{\frac{1}{\beta}}} \sum_{\mathrm{h}=0}^{\infty} \frac{(-\mathrm{k})_{\mathrm{h}}(-1)^{\mathrm{h}}}{h !} \mathrm{e}^{\mathrm{m}+1} \sum_{\mathrm{k}=0}^{\infty} \sum_{\mathrm{m}=0}^{(\lambda \beta(k+1)-1)}\left(\begin{array}{c}
\lambda \beta(k+1)-1 \\
\mathrm{~m}
\end{array}\right)(-1)^{2 \mathrm{~m}} \int_{0}^{\infty} \mathrm{e}^{-\mathrm{z}(\mathrm{m}+1)} z^{\frac{1}{\beta}} \mathrm{dz} \\
B_{k}==\frac{\lambda \beta}{\theta^{\frac{1}{\beta}}} \sum_{\mathrm{h}=0}^{\infty} \frac{(-\mathrm{k})_{\mathrm{h}}(-1)^{\mathrm{h}}}{h !} \mathrm{e}^{\mathrm{m}+1} \sum_{\mathrm{k}=0}^{\infty} \sum_{\mathrm{m}=0}^{(\lambda \beta(k+1)-1)}\left(\begin{array}{c}
\lambda \beta(k+1)-1 \\
\mathrm{~m}
\end{array}\right)(-1)^{2 \mathrm{~m}}\left(\frac{\Gamma\left(\frac{1}{\beta}+1\right)}{(\mathrm{m}+1)^{\frac{1}{\beta}+1}}\right.
\end{gathered}
$$

\subsection{Incomplete Moments}

The incomplete moments play an important role for measuring inequality, for example, income quintiles and Lorenz and Bonferroni curves. These curves depend on the first incomplete moment of the distribution. 
The rth incomplete moment of the LDW family is defined as

$$
\begin{aligned}
M_{r}(y)= & \int_{0}^{y} x^{r}\left(1-\left(\mathrm{e}^{\theta x^{\beta}}-1\right)^{-\lambda}\right)^{-\beta-1}(-1 \\
& \left.+\mathrm{e}^{\theta x^{\beta}}\right)^{-\lambda-1} \mathrm{e}^{\theta x^{\beta}} \theta \lambda \beta^{2} x^{\beta-1} \mathrm{dx}
\end{aligned}
$$

$$
M_{r}(y)=\int_{0}^{y} x^{r} f(x) d x
$$

Using the PDF given in (8), we get

After simplifying we get

$$
M_{r}(y)=\left[\lambda \beta \theta^{k+1} \sum_{n=0}^{\infty} \frac{(a)_{n}}{n !} \sum_{m=0}^{\infty} \frac{(-1)^{m}}{m !}\left(\begin{array}{c}
\lambda(n+1)-1 \\
n
\end{array}\right) \sum_{k=0}^{\infty} \frac{(-1)^{k}}{k !}(n-1)^{k} \frac{q^{r+\frac{1}{\beta}+\frac{k}{\beta}}}{\frac{r}{\beta}+k+1}\right]
$$

The first incomplete moment can be obtained by taking in (15) as $\mathrm{r}=1$.

\subsection{Quantile Points}

The quantile points of LDW distribution are computed by numerically for different sets of values of the parameters as provided in table below by solving the equation

\begin{tabular}{|c|c|c|c|c|c|c|c|}
\hline Parameters & & 0.75 & 0.80 & 0.85 & 0.90 & 0.95 & 0.99 \\
\hline$\theta=1, \beta=0.5, \lambda=1.5$ & $Q_{u}$ & 0.6091 & 0.8158 & 1.1281 & 1.6615 & 2.8493 & 7.1275 \\
\hline$\theta=1, \beta=1.5, \lambda=1.5$ & $Q_{u}$ & 1.2153 & 1.2980 & 1.5382 & 1.7623 & 1.7623 & 2.2477 \\
\hline$\theta=1.5, \beta=1.5, \lambda=1$ & $Q_{u}$ & 1.1064 & 1.2029 & 1.3205 & 1.4763 & 1.7231 & 2.2341 \\
\hline$\theta=0.5, \beta=1.5, \lambda=2$ & $Q_{u}$ & 2.0101 & 2.197224 & 2.4360 & 2.7725 & 3.357517 & 4.7867 \\
\hline$\theta=1, \beta=2, \lambda=2$ & $Q_{u}$ & 1.1246 & 1.1677 & 1.2206 & 1.2914 & 1.4052 & 1.6474 \\
\hline$\theta=2, \beta=1, \lambda=2$ & $Q_{u}$ & 0.5025 & 0.5493 & 0.6090 & 0.6931 & 0.8394 & 1.1966 \\
\hline
\end{tabular}

$$
x=Q_{u}=\left[\frac{1}{\theta}\left(\log \left(u^{-\frac{1}{\beta}}-1\right)^{-\frac{1}{\lambda}}+1\right)\right]^{1 / \beta}
$$

Table 2. Quantile Points of the LDW Distribution

\subsection{Ordered Statistic}

The pdf of the jth order statistic for a random sample of size $\mathrm{n}$ from a distribution function $F(x)$ and an associated $\operatorname{pdf} f(x)$ are given by:

$$
f_{j, n}(x)=\frac{n_{!}}{(j-1)(n-j) !}[F(x)]^{j-1}[1-F(x)]^{n-j} f(x)
$$

where $f(x)$ and $F(x)$ are the pdf and cdf of the LDWD, respectively. The pdf of the jth order statistics for a random sample of size $\mathrm{n}$ from the LDW distribution is, however, given as follows

$$
\begin{gathered}
f_{j, n}(x)=\frac{n !}{(j-1)(n-j) !}\left[\left(1+\left(\mathrm{e}^{\theta x^{\beta}}-1\right)^{-\lambda}\right)^{-\beta}\right]^{j-1}\left[1-\left[\left(1+\left(\mathrm{e}^{\theta x^{\beta}}-1\right)^{-\lambda}\right)^{-\beta}\right]^{n-j}\right. \\
\left(1+\left(e^{\theta x^{\beta}}-1\right)^{-\lambda}\right)^{-\beta-1}\left(-1+e^{\theta x^{\beta}}\right)^{-\lambda-1} e^{\theta x^{\beta}} \theta \lambda \beta^{2} x^{\beta-1}
\end{gathered}
$$

So, the pdf of minimum order statistics is obtained by substituting $j=1$ we have:

$$
f_{j, n}(x)=\frac{n !}{(j-1)(n-j) !}\left[1-\left[\left(1+\left(\mathrm{e}^{\theta x^{\beta}}-1\right)^{-\lambda}\right)^{-\beta}\right]^{n-1}\left(1+\left(\mathrm{e}^{\theta x^{\beta}}-1\right)^{-\lambda}\right)^{-\beta-1}\left(-1+\mathrm{e}^{\theta x^{\beta}}\right)^{-\lambda-1} \mathrm{e}^{\theta x^{\beta}} \theta \lambda \beta^{2} x^{\beta-1}\right.
$$

While the corresponding pdf of maximum order statistics is obtained by making the substitution of $j=n$ in above equation

$$
f_{j, n}(x)=\frac{n !}{(j-1)(n-j) !}\left[\left(1+\left(\mathrm{e}^{\theta x^{\beta}}-1\right)^{-\lambda}\right)^{-\beta}\right]^{n-1}\left(1+\left(\mathrm{e}^{\theta x^{\beta}}-1\right)^{-\lambda}\right)^{-\beta-1}\left(-1+\mathrm{e}^{\theta x^{\beta}}\right)^{-\lambda-1} \mathrm{e}^{\theta x^{\beta}} \theta \lambda \beta^{2} x^{\beta-1}
$$

\section{Entropies}

Entropy is the measure of variation of random variable $X$. The theory of entropy has been used for the characterization of numerous standard probability distributions. Two popular entropy measures are the Shannon and Renyi entropies.

The Shannon entropy of a random variable with p.d.f. $f(x)$ is defined as

$$
S_{H}=-\int_{0}^{\infty} f(x) \log f(x) d x
$$

$$
\log f(x)=-(\beta+1) \ln \left(1+\left(\mathrm{e}^{\theta x^{\beta}}-1\right)^{-\lambda}\right)-(\lambda+1) \ln \left(-1+\mathrm{e}^{\theta x^{\beta}}\right)+\theta x^{\beta}+\ln \left(\theta \lambda \beta^{2}\right)+(\beta-1) \ln \mathrm{x}
$$




$$
S_{H}=(1-\lambda \beta) \int_{0}^{\infty} f(x) \ln \left(\mathrm{e}^{\theta x^{\beta}}-1\right) \mathrm{dx}-\theta \int_{0}^{\infty} f(x) x^{\beta} d x-\ln \left(\theta \lambda \beta^{2}\right) \int_{0}^{\infty} f(x) d x-(\beta-1) \int_{0}^{\infty} f(x) \ln x d x
$$

Where $\int_{0}^{\infty} f(x) d x=1, \int_{0}^{\infty} f(x) \ln x d x=E(\ln x), \int_{0}^{\infty} f(x) x^{\beta} d x=E\left(x^{\beta}\right)$

$$
\begin{gathered}
\ln \left(\mathrm{e}^{\theta x^{\beta}}-1\right)=\sum_{k=0}^{\infty} \frac{(-\theta)^{k} x^{k \beta}}{k !} \\
S_{H}=(1-\lambda \beta) \sum_{\mathrm{k}=0}^{\infty} \frac{(-\theta)^{\mathrm{k}^{\prime} \mu^{\prime} \beta}}{-k !}-\theta E\left(x^{\beta}\right)-\ln \left(\theta \lambda \beta^{2}\right)-(\beta-1) E(\ln x)
\end{gathered}
$$

The Renyi entropy of a random variable $X$ is defined by $I_{k}=\frac{1}{1-k} \log \int_{0}^{\infty}(f(x))^{k} d x$

$$
\begin{gathered}
\left.I_{k}=\frac{1}{1-k} \log \int_{0}^{\infty}\left(\left(1+\left(\mathrm{e}^{\theta x^{\beta}}-1\right)^{-\lambda}\right)^{-\beta-1}\left(-1+\mathrm{e}^{\theta x^{\beta}}\right)^{-\lambda-1} \mathrm{e}^{\theta x^{\beta}} \theta \lambda \beta^{2} x^{\beta-1}\right)\right)^{k} d x \\
I_{k}=\frac{1}{1-\mathrm{k}} \log \left(\theta^{k} \lambda^{k} \beta^{2 k} \sum_{\mathrm{h}=0}^{\infty} \frac{(-\mathrm{k})_{\mathrm{h}}(-1)^{\mathrm{k}}}{h !}\right) \int_{0}^{\infty}\left(1-\mathrm{e}^{\theta x^{\beta}}\right)^{\lambda k(\beta+1))}\left(1-\mathrm{e}^{\theta x^{\beta}}\right)^{-\mathrm{k}(\lambda+1)} \mathrm{e}^{k \theta x^{\beta}} x^{k(\beta-) 1} \mathrm{dx} \\
I_{k}=\frac{1}{1-\mathrm{k}} \log \left(\frac{\theta^{k-1} \lambda^{k} \beta^{2 k-1}}{\theta^{(k-1)\left(1-\frac{1}{\beta}\right)}}\right) \sum_{\mathrm{h}=0}^{\infty} \frac{(-\mathrm{k})_{\mathrm{h}}(-1)^{\mathrm{k}}}{h !} \sum_{\mathrm{k}=0}^{\infty} \sum_{\mathrm{m}=0}^{\mathrm{k}(\lambda \beta-1)}(-1)^{\mathrm{m}} \int_{0}^{\infty} \mathrm{e}^{-\mathrm{z}(\mathrm{m}+\mathrm{k}-1)} z^{\left(1-\frac{1}{\beta}\right)(k-1)} e^{-z} \mathrm{dz} \\
I_{k}=(1-\mathrm{k})^{-1}\left(\frac{1}{\beta}-1\right) \log \theta+k \log \lambda+(2 k-1) \log \beta \log \left(\sum_{\mathrm{h}=0}^{\infty} \frac{(-\mathrm{k})_{\mathrm{h}}(-1)^{\mathrm{k}}}{h !} \sum_{\mathrm{k}=0}^{\infty} \sum_{\mathrm{m}=0}^{\mathrm{k}(\lambda \beta-1)}(-1)^{\mathrm{m}} \frac{\Gamma\left(1-\frac{1}{\beta}\right)(k-1)}{\left.(\mathrm{m}+\mathrm{k}-2)^{\left(1-\frac{1}{\beta}\right)(k-1)}\right)}\right. \\
\text { 6. Bonferroni and Lorenz Curves } E(p)=\frac{1}{p \mu} \int_{0}^{q} x f(x) d x \\
\text { In 1905, Max O. Lorenz represented a model for inequality }
\end{gathered}
$$

\section{Bonferroni and Lorenz Curves} of wealth distribution and C. E. Bonferroni in [1930] proposed a measure of income inequality. Both are used in financial mathematics to check equal distribution of wealth. Bonferroni and Lorenz curves are defined as follows:

Let $X$ be a continuous random variable with probability density function $f(x)$ cumulative distribution function $F(x)$. Let $F^{-1}($.$) denote the quantile function then the Bonferroni$ and Lorenz curves of a random variable $X$ are defined by

And

$$
L(p)=\frac{1}{\mu} \int_{0}^{q} x f(x) d x
$$

Respectively, here $\mu=E(x)$ and $q=F^{-1}(p)$. one can reduce (18) and (19) to

$$
E(p)=\frac{1}{p \mu} \int_{0}^{q} x\left(1-\left(\mathrm{e}^{\theta x^{\beta}}-1\right)^{-\lambda}\right)^{-\beta-1}\left(-1+\mathrm{e}^{\theta x^{\beta}}\right)^{-\lambda-1} \mathrm{e}^{\theta x^{\beta}} \theta \lambda \beta^{2} x^{\beta-1} \mathrm{dx}
$$

After some substitution,

$$
E(p)=\frac{1}{p \mu} \frac{\lambda \beta}{\theta^{\frac{1}{\beta}}} \sum_{n=0}^{\infty} \frac{(a)_{n}}{n !} \int_{0}^{\theta q^{\beta}} \mathrm{z}^{\frac{1}{\beta}}\left(1-\mathrm{e}^{z}\right)^{-\lambda(n+1)-1} e^{\mathrm{z}} \mathrm{dz}
$$

Expanding $\left(1-e^{z}\right)^{-\lambda(n+1)-1}$ by using binomial expansion

$$
\begin{aligned}
& \mathrm{E}(\mathrm{p})=\frac{1}{p \mu} \frac{\lambda \beta}{\theta^{\frac{1}{\beta}}} \sum_{n=0}^{\infty} \frac{(a)_{n}}{n !} \sum_{m=0}^{\infty} \frac{(-1)^{m}}{m !}\left(\begin{array}{c}
\lambda(n+1)-1 \\
n
\end{array}\right) \sum_{k=0}^{\infty} \frac{(-1)^{k}}{k !}(n-1)^{k} \int_{0}^{\theta q^{\beta}} \mathrm{z}^{\frac{1}{\beta}+k} \mathrm{dz} E(p) \\
& E(p)=\frac{1}{p \mu}\left[\lambda \beta \theta^{k+1} \sum_{n=0}^{\infty} \frac{(a)_{n}}{n !} \sum_{m=0}^{\infty} \frac{(-1)^{m}}{m !}\left(\begin{array}{c}
\lambda(n+1)-1 \\
n
\end{array}\right) \sum_{k=0}^{\infty} \frac{(-1)^{k}}{k !}(n-1)^{k} \frac{q^{1+\frac{1}{\beta}+\frac{k}{\beta}}}{\frac{1}{\beta}+k+1}\right]
\end{aligned}
$$

and 


$$
L(p)=\frac{1}{\mu}\left[\lambda \beta \theta^{k+1} \sum_{n=0}^{\infty} \frac{(a)_{n}}{n !} \sum_{m=0}^{\infty} \frac{(-1)^{m}}{m !}\left(\begin{array}{c}
\lambda(n+1)-1 \\
n
\end{array}\right) \sum_{k=0}^{\infty} \frac{(-1)^{k}}{k !}(n-1)^{k} \frac{q^{1+\frac{1}{\beta}+\frac{k}{\beta}}}{\frac{1}{\beta}+k+1}\right]
$$

\section{Characterization of Distribution by Truncated Moments}

In this section, we present our proposed characterizations of three-parameter LDW distribution, with cdf (8) and pdf (9).

Many researchers such as Shakil et al. [28], [29] Ahsanullah et al. [9-11] and Rafique et al. [27] have studied the characterization by truncated moments. We characterize LDW distribution, the first characterization theorem is based on the relation between hazard rate and left truncated moment. The second characterization theorem is based on the relation between reverse hazard rate and right truncated moment. Applying these results, we have characterized LDW distribution by order statistics and upper record values also.

Proposition 1. Suppose the absolutely continuous random variable $Y$ has cdf $F(y)$ with $F(0)=0, F(y)>0 \forall y>$ 0 , p.d.f $f(y)=F^{\prime}(y)$, the

$$
E[Y \mid Y \leq y]=m(y) h(y), y>0
$$

And where,

$$
\begin{gathered}
h(y)=\frac{f(y)}{F(y)} \\
m(y)=\frac{y\left(1-\left[1+\left(e^{\theta x^{\beta}}-1\right)^{-\lambda}\right)\right]^{-\beta}+\int_{0}^{y}\left(1-\left[1+\left(e^{\theta x^{\beta}}-1\right)^{-\lambda}\right)\right]^{-\beta} \mathrm{dt}}{\left[\left(1+\left(e^{\theta x \beta}-1\right)^{-\lambda}\right)^{-\beta-1}\left(-1+e^{\theta x \beta}\right)^{-\lambda-1} e^{\theta x \beta} \theta \lambda \beta^{2} x^{\beta-1}\right]} \\
\text { Proof: } \\
E[Y \mid Y \leq y]=\frac{1}{F(x)} \int_{0}^{y} t f(t) d t \\
m(y) f(y)=\int_{0}^{y} t f(t) d t \\
m(y)=\frac{\int_{0}^{y} t f(t) d t}{f(y)}=\frac{-y\left(1-F(y)+\int_{0}^{y}(1-F(t)) d t\right.}{f(y)}
\end{gathered}
$$

Substituting (8) and (9). Then it is easily seen that

$$
m(y)=\frac{-y\left(1-\left(1+\left(e^{\theta x^{\beta}}-1\right)^{-\lambda}\right)^{-\beta}\right)+\int_{0}^{y}\left(1-\left[1+\left(e^{\theta t^{\beta}}-1\right)^{-\lambda}\right)\right]^{-\beta} \mathrm{dt}}{\left(1+\left(e^{\theta x^{\beta}}-1\right)^{-\lambda}\right)^{-\beta-1}\left(-1+e^{\theta x^{\beta}}\right)^{-\lambda-1} e^{\theta x^{\beta}} \theta \lambda \beta^{2} x^{\beta-1}}
$$

Simple differentiation and simplification gives

$$
m^{\prime}(y)=y-m(y) A(y),
$$

where

$$
A(y)=\frac{f^{\cdot}(y)}{f(y)}=\frac{\theta^{4} \lambda^{2} \beta^{5} x^{4 \beta-5}\left(1-\beta^{2}\right)\left(1+\left(\mathrm{e}^{\theta x^{\beta}}-1\right)^{-\lambda}\right)^{-\beta-2}\left(\mathrm{e}^{\theta x^{\beta}}-1\right)^{-\lambda-1} \beta x^{\beta-1}(\lambda+1)\left(\mathrm{e}^{\theta x^{\beta}}-1\right)^{-\lambda-2} \mathrm{e}^{3 \theta x^{\beta}}}{\left(1+\left(\mathrm{e}^{\theta x^{\beta}}-1\right)^{-\lambda}\right)^{-\beta-1}\left(-1+\mathrm{e}^{\theta x^{\beta}}\right)^{-\lambda-1} \mathrm{e}^{\theta x^{\beta}} \theta \lambda \beta^{2} x^{\beta-1}}
$$

Then we havem ' $(y)=y-m(y)\left\{\frac{\theta^{4} \lambda^{2} \beta^{5} x^{4 \beta-5}\left(1-\beta^{2}\right)\left(1+\left(e^{\theta x^{\beta}}-1\right)^{-\lambda}\right)^{-\beta-2}\left(e^{\theta x^{\beta}}-1\right)^{-\lambda-1} \beta x^{\beta-1}(\lambda+1)\left(e^{\theta x^{\beta}}-1\right)^{-\lambda-2} e^{3 \theta x^{\beta}}}{\left(1+\left(e^{\theta x^{\beta}}-1\right)^{-\lambda}\right)^{-\beta-1}\left(-1+e^{\theta x \beta}\right)^{-\lambda-1} e^{\theta x \beta} \theta \lambda \beta^{2} x^{\beta-1}}\right\}$

From which we obtain $\frac{y-m^{\prime}(y)}{m(y)}=\left\{\frac{\theta^{4} \lambda^{2} \beta^{5} x^{4 \beta-5}\left(1-\beta^{2}\right)\left(1+\left(e^{\theta x^{\beta}}-1\right)^{-\lambda}\right)^{-\beta-2}\left(e^{\theta x^{\beta}}-1\right)^{-\lambda-1} \beta x^{\beta-1}(\lambda+1)\left(e^{\theta x^{\beta}}-1\right)^{-\lambda-2} e^{3 \theta x^{\beta}}}{\left(1+\left(e^{\theta x^{\beta}}-1\right)^{-\lambda}\right)^{-\beta-1}\left(-1+e^{\theta x \beta}\right)^{-\lambda-1} e^{\theta x \beta} \theta \lambda \beta^{2} x^{\beta-1}}\right\}$ we have $\frac{f^{\prime}(y)}{f(y)}=\frac{y-m^{\prime}(y)}{m(y)}$

It follows that

$$
\frac{f^{\cdot}(y)}{f(y)}=\left\{\frac{\theta^{4} \lambda^{2} \beta^{5} x^{4 \beta-5}\left(1-\beta^{2}\right)\left(1+\left(\mathrm{e}^{\theta x^{\beta}}-1\right)^{-\lambda}\right)^{-\beta-2}\left(\mathrm{e}^{\theta x^{\beta}}-1\right)^{-\lambda-1} \beta x^{\beta-1}(\lambda+1)\left(\mathrm{e}^{\theta x^{\beta}}-1\right)^{-\lambda-2} \mathrm{e}^{3 \theta x^{\beta}}}{\left(1+\left(\mathrm{e}^{\theta x^{\beta}}-1\right)^{-\lambda}\right)^{-\beta-1}\left(-1+\mathrm{e}^{\theta x^{\beta}}\right)^{-\lambda-1} \mathrm{e}^{\theta x^{\beta}} \theta \lambda \beta^{2} x^{\beta-1}}\right\}
$$

On integrating the above expression with respect to 'y' and simplifying, we obtain 


$$
\ln f(y)=\ln \left[C\left(1+\left(\mathrm{e}^{\theta x^{\beta}}-1\right)^{-\lambda}\right)^{-\beta-1}\left(-1+\mathrm{e}^{\theta x^{\beta}}\right)^{-\lambda-1} \mathrm{e}^{\theta x^{\beta}} \theta \lambda \beta^{2} x^{\beta-1}\right]
$$

Since $\mathrm{C}$ is determined by $\left.\int_{0}^{\infty} f(y)\right)=1$, we have the pdf.

Proposition 2. Suppose the absolutely continuous random variable $Y$ has $\operatorname{cdf} F(y)$ with

$$
\begin{gathered}
F(0)=0, F(y)>0 \forall y>0 \text {, p.d.f } f(y)=F^{\prime}(y), \text { then } \\
E[Y \mid Y \geq y]=s(y) h(y), y>0
\end{gathered}
$$

And where, $h(y)=\frac{f(y)}{1-F(y)}$

$$
s(y)=\frac{y\left(1-\left[1+\left(e^{\theta x^{\beta}}-1\right)^{-\lambda}\right)\right]^{-\beta}+\int_{\mathrm{y}}^{\infty}\left(1-\left[1+\left(e^{\theta x^{\beta}}-1\right)^{-\lambda}\right)\right]^{-\beta} \mathrm{dt}}{\left[\left(1+\left(e^{\theta x^{\beta}}-1\right)^{-\lambda}\right)^{-\beta-1}\left(-1+e^{\theta x^{\beta}}\right)^{-\lambda-1} e^{\theta x^{\beta}} \theta \lambda \beta^{2} x^{\beta-1}\right]}
$$

Proof:

$$
\begin{gathered}
E[Y \mid Y \geq y]=\frac{1}{1-F(x)} \int_{y}^{\infty} t f(t) d t \\
s(y) f(y)=\int_{y}^{\infty} t f(t) d t \\
s(y)=\frac{\int_{y}^{\infty} t f(t) d t}{f(y)}=\frac{y\left(1-F(y)+\int_{y}^{\infty}(1-F(t)) d t\right.}{f(y)}
\end{gathered}
$$

Substituting (8) and (9). Then it is easily seen that

$$
s(y)=\frac{y\left(1-\left(1+\left(e^{\theta x^{\beta}}-1\right)^{-\lambda}\right)^{-\beta}\right)+\int_{\mathrm{y}}^{\infty}\left(1-\left[1+\left(e^{\theta t^{\beta}}-1\right)^{-\lambda}\right)\right]^{-\beta} \mathrm{dt}}{\left(1+\left(e^{\theta x^{\beta}}-1\right)^{-\lambda}\right)^{-\beta-1}\left(-1+e^{\theta x^{\beta}}\right)^{-\lambda-1} e^{\theta x^{\beta}} \theta \lambda \beta^{2} x^{\beta-1}}
$$

Simple differentiation and simplification gives $s^{\prime}(y)=-y-s(y) A(y)$, where

$A(y)=\frac{f^{\prime}(y)}{f(y)}=\frac{\theta^{4} \lambda^{2} \beta^{5} x^{4 \beta-5}\left(1-\beta^{2}\right)\left(1+\left(\mathrm{e}^{\theta x^{\beta}}-1\right)^{-\lambda}\right)^{-\beta-2}\left(\mathrm{e}^{\theta x^{\beta}}-1\right)^{-\lambda-1} \beta x^{\beta-1}(\lambda+1)\left(\mathrm{e}^{\theta x^{\beta}}-1\right)^{-\lambda-2} \mathrm{e}^{3 \theta x^{\beta}}}{\left(1+\left(\mathrm{e}^{\theta x^{\beta}}-1\right)^{-\lambda}\right)^{-\beta-1}\left(-1+\mathrm{e}^{\theta x^{\beta}}\right)^{-\lambda-1} \mathrm{e}^{\theta x^{\beta}} \theta \lambda \beta^{2} x^{\beta-1}}$

Then we haves' $(y)=-y-s(y)\left\{\frac{\theta^{4} \lambda^{2} \beta^{5} x^{4 \beta-5}\left(1-\beta^{2}\right)\left(1+\left(e^{\theta x^{\beta}}-1\right)^{-\lambda}\right)^{-\beta-2}\left(e^{\theta x^{\beta}}-1\right)^{-\lambda-1} \beta x^{\beta-1}(\lambda+1)\left(e^{\theta x^{\beta}}-1\right)^{-\lambda-2} e^{3 \theta x \beta}}{\left(1+\left(e^{\theta x^{\beta}}-1\right)^{-\lambda}\right)^{-\beta-1}\left(-1+e^{\theta x \beta}\right)^{-\lambda-1} e^{\theta x^{\beta}} \theta \lambda \beta^{2} x^{\beta-1}}\right\}$

From which we obtain $\frac{-y+s^{\prime}(y)}{s(y)}=\left\{\frac{\theta^{4} \lambda^{2} \beta^{5} x^{4 \beta-5}\left(1-\beta^{2}\right)\left(1+\left(e^{\theta x^{\beta}}-1\right)^{-\lambda}\right)^{-\beta-2}\left(e^{\theta x^{\beta}}-1\right)^{-\lambda-1}{ }^{\beta x^{\beta-1}}(\lambda+1)\left(e^{\theta x^{\beta}}-1\right)^{-\lambda-2} e^{3 \theta x^{\beta}}}{\left(1+\left(e^{\theta x \beta}-1\right)^{-\lambda}\right)^{-\beta-1}\left(-1+e^{\theta x \beta}\right)^{-\lambda-1} e^{\theta x \beta} \theta \lambda \beta^{2} x^{\beta-1}}\right\}$

we have $\frac{f^{\prime}(y)}{f(y)}=\frac{-y+s^{\prime}(y)}{s(y)}$

It follows that

$$
\frac{f^{\cdot}(y)}{f(y)}=\left\{\frac{\theta^{4} \lambda^{2} \beta^{5} x^{4 \beta-5}\left(1-\beta^{2}\right)\left(1+\left(\mathrm{e}^{\theta x^{\beta}}-1\right)^{-\lambda}\right)^{-\beta-2}\left(\mathrm{e}^{\theta x^{\beta}}-1\right)^{-\lambda-1} \beta x^{\beta-1}(\lambda+1)\left(\mathrm{e}^{\theta x^{\beta}}-1\right)^{-\lambda-2} \mathrm{e}^{3 \theta x^{\beta}}}{\left(1+\left(\mathrm{e}^{\theta x^{\beta}}-1\right)^{-\lambda}\right)^{-\beta-1}\left(-1+\mathrm{e}^{\theta x^{\beta}}\right)^{-\lambda-1} \mathrm{e}^{\theta x^{\beta}} \theta \lambda \beta^{2} x^{\beta-1}}\right\}
$$

On integrating the above expression with respect to 'y' and simplifying, we obtain 


$$
\ln f(y)=\ln \left[C\left(1+\left(\mathrm{e}^{\theta x^{\beta}}-1\right)^{-\lambda}\right)^{-\beta-1}\left(-1+\mathrm{e}^{\theta x^{\beta}}\right)^{-\lambda-1} \mathrm{e}^{\theta x^{\beta}} \theta \lambda \beta^{2} x^{\beta-1}\right]
$$

Since $\mathrm{C}$ is determined by $\left.\int_{0}^{\infty} f(y)\right)=1$, we have the pdf. Proposition 3: If $X_{1}, X_{2}, \ldots, X_{n}$ be the $n$ independent copies of the random variable $X$ with absolutely continuous distribution function $F(x)$ and pdf $f(x)$, and if $X_{1, n} \leq X_{2, n} \leq \ldots \leq X_{n, n}$ be the corresponding order statistics, then it is known from Ahsanullah et al. [9], chapter 5, or Arnold et al. [12], chapter 2, that $X_{j, n} \mid X_{k, n}=x$, for $1 \leq k<j \leq n$, is distributed as the $(j-k)$ th order statistics from independent observations from the random variable $V$ having the pdf $f_{V}(v \mid x)$ where $f_{V}(v \mid x)=\frac{f(v)}{1-F(x)} \quad, \quad 0 \leq v<x \quad, \quad$ and $X_{i, n} \mid X_{k, n}=x, 1 \leq i<k \leq n$, is distributed as ith order statistics from $k$ independent observations from the random variable $W$ having the pdf $f_{W}(w \mid x)$ where $f_{W}(w \mid x)=\frac{f(w)}{F(x)}, w<x$ Let

$S_{k-1}=\frac{1}{k-1}\left(X_{1, n}+X_{2, n}+\ldots+X_{k-1, n}\right)$ and $T_{k, n}=\frac{1}{n-k}\left(X_{k+1, n}+X_{k+2, n}+\ldots+X_{n . n}\right)$. Now, suppose the random variable $X$ is absolutely continuous with the cumulative distribution function $F(x)$ and the probability density function $f(x)$. We assume that $\omega=\inf \{x \mid F(x)>0\}$, and $\delta=\sup \{x \mid F(x)<1\}$. We also assume that $f(x)$ is a differentiable for all $x$, and $E(X)$ exists. Taking $\omega=0$ and $\delta=\infty$, we have $E\left(S_{k-1} \mid X_{k, n}=x\right)=m(x) h(x)$, where $m(x)$ and $h(x)$ are respectively given by the expressions in Proposition 1 , if and only if $X$ has the pdf(9).

Proof: It is known that $E\left(S_{k-1} \mid X_{k, n}=x\right)=E(X \mid X \leq x)$; see Ahsanullah et al. [9], and David and Nagaraja [18]. Hence, by Proposition 1, the result follows.

Proposition 4. Suppose the random variable $X$ is absolutely continuous with the cumulative distribution function $F(x)$ and the probability density function $f(x)$. We assume that $\omega=0$ and $\delta=\infty$. We also assume that $f(x)$ is a differentiable for all $x$, and $E(X)$ exists. Then
$E\left(T_{k, n} \mid X_{k, n}=x\right)=s(x) h(x)$, where $s(x)$ and $h(x)$ are respectively given by the expressions in Proposition 2, if and only if $X$ has the pdf (9).

Proof: Since $E\left(T_{k, n} \mid X_{k, n}=x\right)=E(X \mid X \geq x)$, see Ahsanullah et al. [9], and David and Nagaraja [18], the result follows from Proposition 2.

Proposition 5. Let be a sequence of independent and identically distributed absolutely continuous random variables with distribution function $F(x)$ and pdf $f(x)$. If $Y_{n}=\max \left(X_{1}, X_{2}, \ldots, X_{n}\right)$ for $n \geq 1$ and $Y_{j}>Y_{j-1}, j>1$, then $X_{j}$ is called an upper record value of $\left\{X_{n}, n \geq 1\right\}$. The indices at which the upper records occur are given by the record

$\left\{U(n)>\min \left(j \mid j>U(n+1), X_{j}>X_{U(n-1)}, n>1\right)\right\} \quad$ and $U(1)=1$. Let the nth upper record value be denoted by $X(n)=X_{U(n)}$. For details on record values, see Ahsanullah [8].

Now, Suppose the random variable $X$ is absolutely continuous with the cumulative distribution function $F(x)$ and the probability density function $f(x)$. We assume that $\omega=0$ and $\delta=\infty$. We also assume that $f(x)$ is a differentiable for all $x$, and $E(X)$ exists. Then $E(X(n+1) \mid X(n)=x)==s(x) h(x)$, where $s(x)$ and $h(x)$ are respectively given by the expressions in Proposition 2, if and only if $X$ has the pdf (9).

Proof: It is known from Ahsanullah et al. [8] and Nevzorov [24] that $E(X(n+1) \mid X(n)=x)=E(X \mid X \geq x)$. Then, the result follows from Proposition 2.

\section{Maximum Likelihood Estimation}

Several approaches for parameter estimation have been proposed in the literature but the maximum likelihood method is the most commonly employed.

Let $X_{1}, X_{2}, \ldots, X_{m}$ be a random sample of size $\mathrm{n}$ of the LDW distribution then the total log-likelihood (LL) function is given

$$
\begin{aligned}
& \quad \mathrm{L}(\lambda, \theta, \beta)=\beta^{2 n} \prod_{i=1}^{n}\left(1+\left(e^{\theta x^{\beta}}-1\right)^{-\lambda}\right)^{-\beta-1} \lambda^{n} \prod_{i=1}^{n}\left(-1+e^{\theta x^{\beta}}\right)^{-\lambda-1} \theta^{n} \prod_{i=1}^{n} e^{\theta x^{\beta}} \prod_{i=1}^{n} x^{\beta-1} L(\lambda, \theta, \beta) \\
& =2 n \log [\beta]+n \log [\theta]+n \log [\lambda]+\theta \sum_{I=1}^{n} x_{I}^{\beta}+(\beta-1) \sum_{I=1}^{n} \log \left[x_{I}\right]-(\lambda+1) \sum_{I=1}^{n} \log \left[e^{x_{I}{ }^{\beta} \theta}-1\right]+\lambda(\beta \\
& \quad+1) \sum_{I=1}^{n} \log \left(1+\left(e^{x_{I}{ }^{\beta} \theta}-1\right)\right)
\end{aligned}
$$

The First derivatives of the log-likelihood function are given as follow 


$$
\begin{gathered}
\frac{\delta L(\lambda, \theta, \beta)}{\delta \beta}=\frac{2 n}{\beta}+\theta \beta \sum_{I=1}^{n} x_{I}^{\beta-1}+\sum_{I=1}^{n} \log \left[x_{I}\right]-(\lambda+1) \sum_{I=1}^{n} \theta \beta x_{I}{ }^{\beta-1}+2 \lambda \sum_{I=1}^{n} \beta x_{I}{ }^{\beta-1} \theta \\
\frac{\delta L(\lambda, \theta, \beta)}{\delta \lambda}=\frac{n}{\lambda}-\sum_{I=1}^{n} \log \left[\mathrm{e}^{x_{I} \beta}-1\right]+\lambda(\beta+1) \sum_{I=1}^{n} \log \left(1+\left(\mathrm{e}^{x_{I} \beta}-1\right)\right) \\
\frac{\delta L(\lambda, \theta, \beta)}{\delta \theta}=\frac{n}{\theta}+\sum_{I=1}^{n} x_{I}{ }^{\beta}-(\lambda+1) \sum_{I=1}^{n} x_{I} \beta+\lambda(\beta+1) \sum_{I=1}^{n} x_{I}^{\beta}
\end{gathered}
$$

Equating equations to zero and solving them numerically, one can obtain the estimates of the unknown parameters.

\section{Simulation Study}

This section deals the simulation study. In proposed model we generated random variables by using CDF of LDWD with four different value of parameters for $\mathrm{n}=25,50,100$. Parameters are estimated with method of MLE by using each generated random variable.

In statistical study, bias states to the tendency of a measurement process to over or under estimate the value of population parameters. Squared error is a function which obtained from square values of bias. MSE is always constructive. Bias shows the contrasts between estimated values of parameter variation from true value of parameter.
By using the estimated parameters, we calculated Bias and MSE of LDWD. All simulations were done on computational software 'Mathematica.

The analysis computes the coming values:

Average bias of the simulated estimates:

$$
\frac{1}{1000} \sum_{i=1}^{1000}\left(\Theta^{*}-\Theta\right)
$$

Average mean square error (MSE) of the simulated estimates:

\begin{tabular}{|c|c|c|c|c|c|c|}
\hline $\bar{n}$ & $\operatorname{Bias}(\widehat{\theta})$ & $\operatorname{Bias}(\widehat{\boldsymbol{\beta}})$ & $\operatorname{Bias}(\hat{\lambda})$ & $\operatorname{MSE}(\widehat{\boldsymbol{\theta}})$ & $\operatorname{MSE}(\widehat{\boldsymbol{\beta}})$ & $\operatorname{MSE}(\hat{\lambda})$ \\
\hline 25 & 0.65234 & -0.08837 & 0.56572 & 0.56572 & 0.40559 & 0.86286 \\
\hline 50 & 0.35310 & 0.23613 & 0.17950 & 0.29754 & 0.52918 & 0.15562 \\
\hline 100 & 0.27740 & 0.46278 & -0.01169 & 0.11882 & 0.72056 & 0.04713 \\
\hline
\end{tabular}

$$
\frac{1}{1000} \sum_{i=1}^{1000}\left(\Theta^{*}-\Theta\right)^{2}
$$

The results are reported in Tables 3 and 4.

Table 3. Average mean of Bias and MSE values for estimators $\widehat{\theta}, \hat{\beta}$ and $\hat{\lambda}$ of data 1 .

Table 4. Average mean of Bias and MSE values for estimators $\widehat{\theta}, \hat{\beta}$ and $\hat{\lambda}$ of data 2.

\begin{tabular}{lllllll}
\hline $\boldsymbol{n}$ & $\operatorname{Bias}(\widehat{\boldsymbol{\theta}})$ & $\operatorname{Bias}(\widehat{\boldsymbol{\beta}})$ & Bias $(\widehat{\boldsymbol{\lambda}})$ & MSE $(\boldsymbol{\theta})$ & MSE $(\widehat{\boldsymbol{\beta}})$ & 0.25397 \\
\hline 25 & 1.00745 & -0.44500 & -0.34131 & 1.26654 & 0.21862 & 0.09400 \\
10 & 0.57483 & -0.23206 & -0.39724 & 0.39676 & 0.19138 & 0.63077 \\
\hline
\end{tabular}

\section{Evaluation Measures and Practical Data Examples}

In this section, we illustrate the usefulness of the $\log$ dagum weibull distribution and compare the results with the weibull distribution, gamma distribution, Lomax distribution, exponentiated exponential distribution, Nadarajah exponentiated exponential distributions by means of four real data sets. We will check goodness of fit of our model with some test statistics like AD test, CVM test, K-S test and pvalue. All calculations are executed on computational software MATHEMATICA.

In order to demonstrate the proposed methodology, we consider four different practical data sets described below with their analysis. Moreover, perfection of competing models is also tested via the Kolmogrov-Simnorov (K S), the Anderson Darling $\left(A^{*}\right)$ and the Cramer-von Misses $\left(W^{*}\right)$ statistics. The mathematical expressions for the statistics are given by

$$
\begin{aligned}
K S & =\max \left\{\frac{i}{m}-z_{i}, z_{i}-\frac{i-1}{m}\right\} \\
\mathrm{A}^{*} & =\left(\frac{2.25}{\mathrm{~m}^{2}}-\frac{0.75}{\mathrm{~m}}+1\right)\{-1 \\
& \left.-\frac{1}{\mathrm{~m}} \sum_{1}^{m}(2 \mathrm{i}-1) \ln \left(\mathrm{z}_{\mathrm{i}}\left(\mathrm{z}_{\mathrm{m}-\mathrm{i}+1}\right)\right)\right\} \\
\mathrm{w}^{*} & =\sum_{1}^{\mathrm{m}}\left(\mathrm{z}_{\mathrm{i}}-\frac{2 \mathrm{i}-1}{2 \mathrm{~m}}\right)^{2}+\frac{1}{12 \mathrm{~m}}
\end{aligned}
$$

Data set 1 . Thefirstdata set of leukemia-free survival times of 50 patients with Autologous transplant. Data sets are presented in the following tables $0.03,0.493,0.855,1.184$, $1.283,1.480,1.776,2.138,2.500,2.763,2.993,3.224,3.421$, 4.178, 4.441, 5.691, 5.855, 6.941, 6.941, 7.993, 8.882, 8.882, $9.145,11.480,11.513,12.105,12.796,12.993,13.849$, $16.612,17.138,20.066,20.329,22.368,26.776,28.717$, 
$28.717,32.928,33.783,34.211,34.770,39.539,41.118, \quad 45.033,46.053,46.941,48.289,57.401,58.322,60.625$.

Table 5. AD, CVM, The K-S statistics and p-values for the data set 1.

\begin{tabular}{lllll}
\hline Distributions & $\boldsymbol{A}^{*}$ & $\boldsymbol{W}^{*}$ & K-S & p-value \\
\hline LDWD & 0.403996 & 0.0651719 & 0.076948 \\
EED & 0.362828 & 0.0483839 & 0.084435 \\
WD & 0.411538 & 0.0562415 & 0.0868536 \\
GD & 0.369975 & 0.0496265 & 0.0847622 & 0.868171 \\
LD & 2.504843 & 0.3799524 & 0.19666206 & 0.86513 \\
NEED & 0.666096 & 0.0962511 & 0.0906376 & 0.04182 \\
\hline
\end{tabular}

Table 6. Information Criteria of Different Distributions for Data 1.

\begin{tabular}{|c|c|c|c|c|c|}
\hline Distributions & AIC & AICC & BIC & HQIC & CAIC \\
\hline LDWD & 394.682 & 395.140 & 398.235 & 396.37 & 395.140 \\
\hline EED & 394.954 & 395.209 & 398.778 & 396.41 & 395.209 \\
\hline WD & 395.433 & 395.689 & 399.257 & 396.89 & 395.689 \\
\hline LD & 394.783 & 395.304 & 400.5187 & 396.97 & 395.304 \\
\hline NEED & 396.045 & 396.301 & 399.869 & 397.50 & 396.301 \\
\hline
\end{tabular}

Data set 2: Second data set Lifetime of 50 devices is 0.1 , $0.2,1,1,1,1,1,2,3,6,7,11,12,18,18,18,18,18,21,32$, $36,40,45,46,47,50,55,60,63,63,67,67,67,67,72,75$, $79,82,82,83,84,84,84,85,85,85,85,85,86,86$.

We fit the LDW model and other competitive models such as the Exponentiated exponential distribution (EED), Weibull distribution (WD), Gamma distribution (GD), NEED Nadarajah Exponentiated exponential distribution and Lomax distribution (LD) to data sets.

Table 7. The K-S statistics and p-values for the data set 2.

\begin{tabular}{lllll}
\hline Distributions & $\boldsymbol{A}^{*}$ & $\boldsymbol{W}^{*}$ & K-S & p-value \\
\hline LDWD & 0.41395 & 0.06328 & 0.07134 & 0.9135 \\
EED & 0.36282 & 0.04838 & 0.08444 & 0.8682 \\
WD & 0.41153 & 0.05624 & 0.08685 & 0.8450 \\
GD & 0.36997 & 0.04962 & 0.08476 & 0.8651 \\
LD & 8.09533 & 1.66869 & 0.3377 & 0.00002 \\
NEED & 8.11488 & 1.67229 & 0.322722 & 0.00006 \\
\hline
\end{tabular}

Table 8. Information Criteria of Different Distributions for Data 2.

\begin{tabular}{lllll}
\hline Model & AIC & AICC & BIC & CAIC \\
\hline LDWD & 455.064 & 455.586 & 460.800 & 455.586 \\
EED & 483.99 & 484.246 & 487.814 & 484.246 \\
WD & 486.004 & 486.259 & 489.828 \\
GD & 484.38 & 484.636 & 488.204 & 486.259 \\
NEED & 516.033 & 519.857 & 516.289 & 484.636 \\
LD & 474.0873 & 474.3427 & 477.9114 & 516.857 \\
\hline
\end{tabular}

Data set 3: This data set consists of 100 uncensored data on breaking stress of carbon fibres (in Gba), 0.39, 0.81, 0.85, $0.98,1.08,1.12,1.17,1.18,1.22,1.25,1.36,1.41,1.47,1.57$, $1.57,1.59,1.59,1.61,1.61,1.69,1.69,1.71,1.73,1.8,1.84$, $1.84,1.87,1.89,1.92,2,2.03,2.03,2.05,2.12,2.17,2.17$, $2.17,2.35,2.38,2.41,2.43,2.48,2.48,2.5,2.53,2.55,2.55$, $2.56,2.59,2.67,2.73,2.74,2.76,2.77,2.79,2.81,2.81,2.82$, $2.83,2.85,2.87,2.88,2.93,2.95,2.96,2.97,2.97,3.09,3.11$, $3.11,3.15,3.15,3.19,3.19,3.22,3.22,3.27,3.28,3.31,3.31$, $3.33,3.39,3.39,3.51,3.56,3.6,3.65,3.68,3.68,3.68,3.7$,
$3.75,4.2,4.38,4.42,4.7,4.9,4.91,5.08,5.56$.

Table 9. Information criteria of different distributions for data 3.

\begin{tabular}{llllll}
\hline Model & AIC & BIC & AICC & HQIC & CAIC \\
\hline LDWD & 288.62 & 296.43 & 288.8685 & 296.4883 & 296.4883 \\
GD & 290.4673 & 295.6775 & 290.5909 & 292.576 & 290.5909 \\
WD & 289.06 & 296.87 & 289.3086 & 292.2217 & 289.3086 \\
EED & 296.36466 & 301.574 & 296.488 & 298.4733 & 296.4883 \\
NEED & 393.8472 & 399.0575 & 393.9709 & 395.9559 & 393.9709 \\
LD & 474.0873 & 477.9114 & 474.3427 & 475.54356 & 474.3427 \\
\hline
\end{tabular}

Table 10. The K-S statistics and p-values for the data sets 3.

\begin{tabular}{llll}
\hline Distributions & $\boldsymbol{A}^{*}$ & $\boldsymbol{W}^{*}$ & K-S \\
\hline LDWD & 0.39666 & 0.06508 & 0.0618 \\
EED & 1.2341 & 0.2303 & 0.1077 \\
WD & 18.9521 & 3.7772 & 0.3341 \\
GD & 200.5016 & 32.9885 & 0.9996 \\
LD & 79.3018 & 17.3623 & 0.8210 \\
NEED & 16.9307 & 3.35163 & 0.3170 \\
\hline
\end{tabular}


Data set 4: This data consist times to failure of eighteen electronic devices used to show how the proposed distribution can be appliedin practice. $5,11,21,31,46,75,98,122,145,165,196,224,245,293,321,330,350,420$.

Table 11. The K-S statistics and p-values for the data sets 4.

\begin{tabular}{llll}
\hline Distributions & $\boldsymbol{A}^{*}$ & $\boldsymbol{W}^{*}$ & K-S \\
\hline LDWD & 0.1725 & 0.02361 & 0.0840 \\
EED & 0.4456 & 0.07077 & 0.12138 \\
WD & 0.4609 & 0.0644 & 0.1132 \\
GD & 0.4487 & 0.06986 & 0.1206 \\
LD & 28.2328 & 5.0981 & 0.9157 \\
NEED & 2.46950 & 0.4826 & 0.9535 \\
\end{tabular}

Table 12. Information Criteria of Different Distributions for Data 4.

\begin{tabular}{|c|c|c|c|c|c|}
\hline Model & AIC & BIC & HQIC & AICC & CAIC \\
\hline LDWD & 208.2915 & 210.9626 & 208.6598 & 210.0058 & 210.0057 \\
\hline GD & 226.1 & 227.9 & 226.9 & 229.9 & 229.9 \\
\hline WD & 395.433 & 397.214 & 395.6789 & 396.233 & 396.233 \\
\hline NEED & 237.8595 & 239.6403 & 238.10512 & 238.6596 & 238.65956 \\
\hline LD & 341.4154 & 343.1962 & 341.6609 & 342.2154 & 342.2154 \\
\hline
\end{tabular}

\section{Analysis}

The measures of goodness of fit including the Akaike information criterion (AIC), consistent Akaike information criterion (CAIC), Bayesian information criterion (BIC), Hannan-Quinn information criterion (HQIC), AndersonDarling $\left(A^{*}\right)$, Cram'er-von Mises $\left(W^{*}\right)$ and KolmogrovSmirnov (K-S) statistics are computed to compare the fitted models. In general, the smaller values of these statistics better fit to the data. The required computations are carried out in the Mathematica 11.0.

Tables 4 to 12 represents that the LDWD model produces the heigh $\mathrm{p}$-value and the smallest test statistics value and therefore fitted better than the others (WD, GD, LD, EED, NEED) for the estimated parameter the pdf of the distributions have been superimposed on the histogram of four data sets provided as figures 6,7 and 8 .

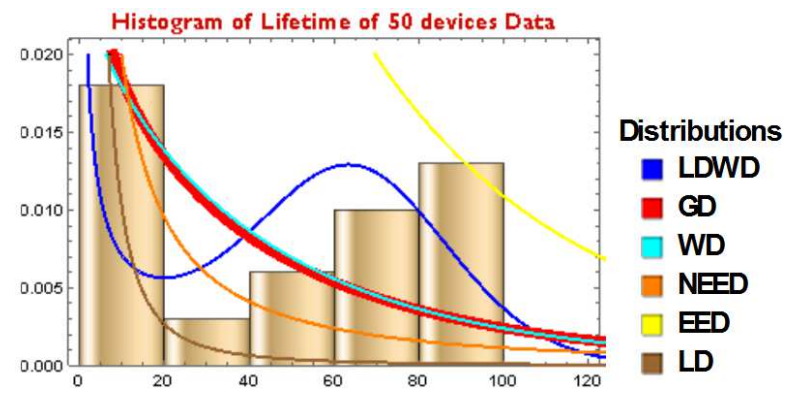

Figure 6. Fitted densities for data 1 .

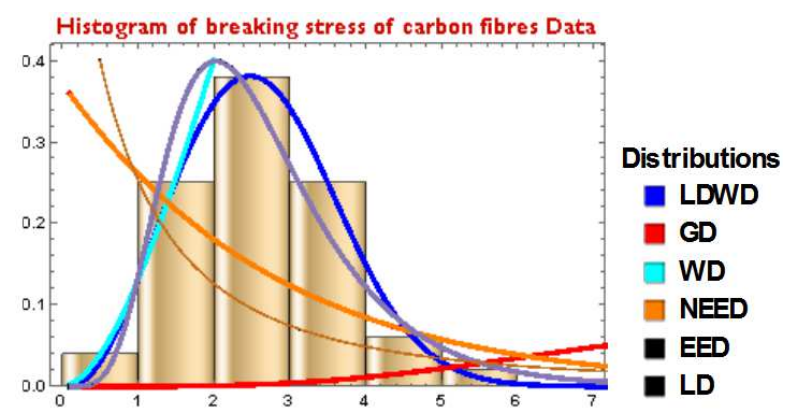

Figure 7. Fitted densities for data 2.

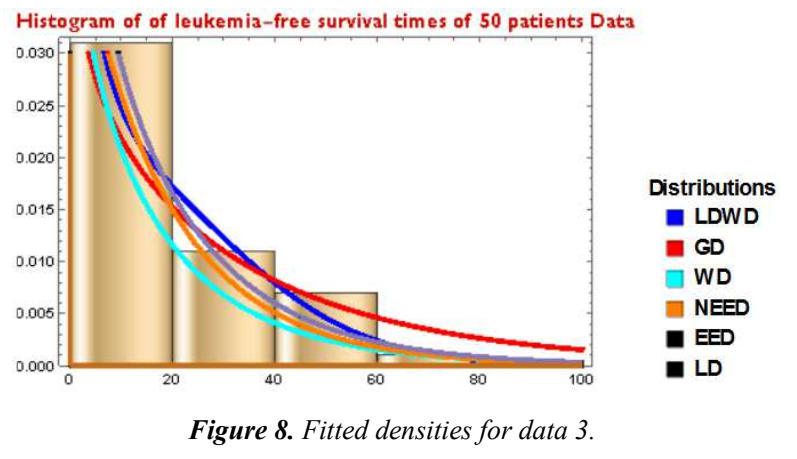

\section{Conclusion}

There has been an increased interest in defining new generated classes of univariate continuous distributions. The extended distributions have attracted several statisticians to develop new models. Current article proposes the new Log-Dagum T-X family of distributions. Some of its mathematical properties have been derived; estimation of the parameters has been discussed. Also, computations of Quantile points and Characterizations have been done. The application of LDW distributions has been studied using four real life-time data sets. It has been observed that the proposed special model is consistently better fit than other competing models. It is expected that this new family and its generated models will attract wider application in several areas such as engineering, survival and lifetime data, hydrology, economy.

\section{References}

[1] Alzaghal, A., Lee C., and Famoye, C. F. (2013). Exponentiated T-X family of distributions with some applications. International Journal of Probability Statistics, 2, 31-49.

[2] Ahmad, Z.(2018). A new generalized class of distributions properties and estimation based on type I censored samples. Annals of Data Science https://doi.org/10.1007/s40745-0180160-5. 
[3] Alzaatreh, A., Famoye, F., and Lee, C. (2013). A new method for generating families of continuous distributions. Metron, 71, 63-79.

[4] Alzaatreh, A., Famoye, F. and Lee, C. (2014). The gammanormal distribution properties and applications. Computational statistics and data analysis, 69, 67-80.

[5] Alexander, C., Cordeiro, G., Ortega, E., and Sarabia, C. (2012). Generalized beta- generated distributions. Computational statistics and data analysis, 56, 1880-1897.

[6] Aslam, M., Asghar, Z., Hussain, Z., and Farooq, S. S. (2020) A modified T-X family of distributions classical and bayesian analysis. Journal of Taibah University for Science, $1,254-264$.

[7] Amini, M., Mir Mostafaee, S. J., and Ahmadi, J. (2014). Loggamma-generated families of distributions. Statistics, 2014, 748-775.

[8] Ahsanullah, M. (1995). Record Statistics, Nova Science Publishers, New York, USA.

[9] Ahsanullah, M., Nevzorov, V. B., and Shakil, M. (2013a). An Introduction to Order Statistics Press, Paris, France.

[10] Ahsanullah, M., Shakil, M., and Kibria, B. (2016). Characterizations of Continuous Distributions by Truncated Moment, Journal of Modern Applied Statistical Methods, 1, 316-331.

[11] Ahsanullah, M., (2017). Characterization of Univariate Continuous Distributions, Atlantis-Press, Paris, France.

[12] Arnold, B. C., Balakrishnan, and Nagaraja, H. N., (2005). First Course in Order Statistics Wiley, New York, USA.

[13] Bourguignon, M., Silva, R., and Cordeiro, G., (2014). The Weibull-G family of probability distribution. Journal of data Science, 12, 53-68.

[14] Cordeiro, G., de Castro, M., (2011). A new family of generalized distributions. Computational statistics and data analysis, $81,883-893$.

[15] Cordeiro, G., Ortega, E., Da Cunha, D., (2013). The exponentiated generalized class of distributions. Journal of data Science, 11, 1-27.

[16] Cordeiro, G. M., Alizadeh, M., and Diniz, M. P. R., (2016). The type I half-logistic family of distributions. Journal of statistical computation and simulation, 86, 707-728.

[17] Cordeiro, G. M., Alizadeh, M., and Ortega, E. M. M. (2014). The exponentiated half-logistic family of distribution properties and applications. Journal of probability and statistics.

[18] David, H. A., and Nagaraja, H. N. (2003). Order Statistics, Third Edition, Wiley, New York, USA.

[19] Eugene, N., Lee, C., and Famoye, F. (2002). Beta-normal distribution and its applications. Communications in statistics Theory and Methods, 31, 497-512.
[20] Jamal, F., and Nasir, M. (2019). Some new members of The T$\mathrm{X}$ family of distributions. 17th International conference on statistical sciences Lahore, Pakistan, hal-01965176v3.

[21] Hamed, D., and Alzaghal, A. (2021). New Class of Lindley Distribution. J. Stat. Distrib. App 8, 11.

[22] Handique, L., Akbar, M., Mohsin, M., and Jamal, F. (2021). Properties and applications of a new member of the T-X family of distributions. Thailand statistician, 19, 248-260.

[23] Hassan, A. S., Elgarhy, M. (2016). Kumaraswamy weibullgenerated family of distributions with applications. Advances and applications in statistics, 48, 205-239.

[24] Nevzorov, V. B. (2001). Records: Mathematical TheoryTranslation of Mathematical Monograph, American Mathematical Society, Rhode Island, USA.

[25] Nasiru, S., Mwita, N. P., and Ngesa, O. (2017). Exponentiated generalized exponential dagum distribution, Journal of King Saud University - Science, 31, 362-371.

[26] Ristic, M., Balakrishnan N. (2012). The gamma-exponentiated exponential distribution. Journal of Statistical Computation and Simulation, 82, 1191-1206.

[27] Rafique, A., and Saud, N. (2021). Some Characterizations of Unimodal, Reserve J-Shaped Distribution. 18th International Conference on Statistical Sciences Lahore, Pakistan, 35, 1820 .

[28] Shakil, M., Ahsanullah, M., and Kibria, B. M. (2014). A new characterization of skew normal distribution by truncated moment. Applications and Applied Mathematics, 9, 28-38.

[29] Shakil, M., Ahsanullah, M., and Kibria, B. M. (2018). On the Characterizations of Chen's Two-Parameter Exponential Power Life-Testing Distribution. Journal of Statistical Theory and Applications, 3, 393-407.

[30] Shakil, M., Kibria, B. M., and Ahsanullah M. (2021). Some Inferences on Dagum (4P) Distribution, Statistical Distribution and their Applications, 154, 1-33.

[31] Shakil, M., Kibria, B. M., Singh, J. N., and Ahsanullah M. (2021). On Burr (4P) Distribution Application of Breaking Stress Data, Statistical Distributions and their Applications, 2, 190-199.

[32] Torabi, and Montazari, N. (2014). The logistic-uniform distribution application. Communications in Statistics Simulation and Computation, 43, 2551-2569.

[33] Torabi, Montazari, N., (2012). The gamma-uniform distribution and its application. Kybernetika, 48, 16-30.

[34] Tahir, M. H., Zubair, M., Mansoor, M., Cordeiro, G. M., Alizadeh, M., and Hamedani, G. H. (2016). A new Weibull-G family of distributions. Hacettepe Journal of Mathematics and Statistics, 45, 629-647.

[35] Zografos, K., and Balakrishnan, N. (2009). On families of beta- and generalized gamma- generated distributions and associated inference. Stat. Methodology 6, 344-362. 